\begin{tabular}{|c|l|}
\hline Title & Cantor coding in the hippocampus \\
\hline Author(s) & Tsuda, Ichiro; Kuroda, Shigeru \\
\hline Citation & Japan Journal of Industrial and A pplied Mathematics, 18(2), 249_258 \\
\hline https://doi.org/_0.1007/BFO3168573 \\
\hline Issue Date & 2001 \\
\hline Doc URL & http://hdl.handle.net/2115/47071 \\
\hline Rights & The original publication is available at www.springerlink.com \\
\hline Type & article (author version) \\
\hline File Information & Tsudal-Kurodas_2001_HUSCAP_2.pdf \\
\hline
\end{tabular}

Instructions for use 


\title{
Cantor coding in the hippocampus
}

\author{
Ichiro Tsuda and Shigeru Kuroda \\ Applied Mathematics and Complex Systems Research Group, \\ Department of Mathematics, Graduate School of Science, \\ Hokkaido University, \\ Sapporo, 060-0810 Japan
}

\begin{abstract}
We construct a mathematical model for the dynamic behavior of hippocampus. The model is described by the skew product transformation in terms of chaotic dynamics and contracting dynamics. In the contracting subspace, fractal objects are generated. We show that such fractal objects are characterized by a code of a temporal sequence generated by chaotic dynamics.
\end{abstract}

Key words: Cantor coding, fractal, chaotic dynamics, skew product transformation, hippocampus 


\section{Introduction}

Our aim in this paper is to show how information of the orbits generated by chaotic dynamical system is encoded in Cantor sets generated in a contracting subspace in chaos-driven contracting systems, and also to apply this idea to the construction of a mathematical model for the dynamic behavior of hippocampus in the brain. A type of coding that we treat in this paper is a kind of temporal coding [1]. The hippocampus is known to be responsible for the formation of episodic memory. Without hippocampus no one can transform short-term memories to long-term memories. Thus the present study may provide a mathematical basis of the mechanism for the formation of episodic memory [2].

In our mathematical model of hippocampus, we treat, in particular, the unidirectional coupling from $\mathrm{CA} 3$ to $\mathrm{CA} 1$, both of which also receive the control signal from the septum. Here, from the phisiological and anatomical (structural) reason, it is proper to assume that CA3 is an unstable network which can generate chaotic behavior and CA1 is a stable network in the case without synaptic modifications. We found a Cantor set in the phase space of the model CA1, which is generated, driven by chaotic behavior in the model CA3. From the mathematical point of view, the problem is thus how information of the orbits (or symbol sequence) generated by chaotic dynamics is encoded in a Cantor set generated in the contracting subspace in the chaos-driven contracting system.

It is known that a coding on a Cantor set is realized in iterated function system (IFS) $[3,4,5,6]$ and also in recurrent neural network (RNN) [7, 8, 9, 10]. It is, however, not known how to encode the information of symbol sequence generated in chaotic dynamical systems into its subspace. We discuss this issue in several chaos-driven contracting systems, where various Cantor 
sets $[11,12,13]$ are the subject for the study.

In $\S 2$, we show a mechanism of Cantor coding by using simple dynamical systems. A mathematical model of the hippocampus is presented in $\S 3$. The Candor coding in the hippocampal model is presented in $\S 4$. Section 5 is devoted to summary and discussion.

\section{A typical chaos-driven contracting system}

In this section, we present a simple example of chaos-driven contracting system. A dissipative baker's map is presented, which can be viewed as a skew product transformation $T(x, y)=\left(f(x), g_{x}(y)\right)$ with a chaotic mapping $f$ and a contracting mapping $g$.

The following equations describe a dissipative baker's map.

For $x, y \in R, t \in N$, and $\mu<\frac{1}{2}$,

$$
\begin{aligned}
& x(t+1)=2 x(t) \quad(\bmod 1), \\
& y(t+1)=\left\{\begin{array}{l}
\mu y(t) \quad\left(x(t) \leq \frac{1}{2}\right) \\
\mu y(t)+1-\mu \quad\left(x(t)>\frac{1}{2}\right) .
\end{array}\right.
\end{aligned}
$$

Here, $t$ indicates a discrete time and $\mu$ a dissipation parameter. The variable $x$ constitutes chaotic dynamics by itself. Actually, it provides a Bernoulli shift. On the other hand, the variable $y$ constitues contracting dynamics in the present $\mu$ 's condition, the dynamics of which depend on the $x$ variable. Thus the (dissipative) baker's map is considered to be a skew product transformation in the sense of a chaos-driven contracting system. In the contracting subspace $y$, a Cantor set is generated. Actually, one can "observe" a Cantor set on any crosssection, $x=$ constant, which is taken not to see the chaotic behavior.

When we label the chaotic orbits arriving in the subintervals $x \leq \frac{1}{2}$ and $x>\frac{1}{2}$ by, for instance, " 0 " and " 1 ", respectively, we say that the Bernoulli 
shift generates symbol sequence consisting of 0 and 1 . This kind of chaotic symbol sequence is encoded in the Cantor set created in the contraction subspace. A characteristic of encoding is as follows. An itinerary of the sequence is hierarchically encoded in the Cantor set. In other words, a temporal structure of the sequence is encoded in a spatial (or geometric) hierarchy of the Cantor set. Generalizing this scheme, we wish to call it a Cantor coding. There are many other chaotic dynamical systems like the solenoid which can also be written in the form of a skew product transformation.

\section{A model for the hippocampus}

The model we wish to propose is a kind of macroscopic model at the network level. In other words, we are concerned with an averaged activity of neuron, thus our network consists of several peculiar network components, based on the neuron model representing a simple input-output function such as a sigmoid function. In order to construct a model for the hippocampus, let us, for the first time, describe the physiological and anatomical facts related to the network. The hippocampus consists of several areas. Among others, the network within areas called CA3 and CA1, and its inter-areal connections have been investigated.

In CA3, the axon-collaterals of the pyramidal cells make the synaptic contacts with themselves, and construct recurrent connections. Thus the pyramidal cells in CA3 constitute, so called a recurrent network. Furthermore, each pyramidal cell makes a synaptic contact with a neighboring inhibitory cell. Such an inhibitory cell excited by the outputs of pyramidal cell inhibits the neighboring pyramidal cells via the synaptic contacts. The other axon-collaterals of the pyramidal cells called Schaffer collaterals make synaptic contacts with the apical dendrites of the pyramidal cells in CA1. 
This type of connections constitute a unidirectional coupling of the CA3 network with CA1 network. Mathematically, this can be called a skew-product transformation of the dynamics in CA3 and CA1.

In CA1, the recurrent connections among pyramidal cells have not been clearly found. The inhibitory cells exist also in CA1, each of which receives mainly the output of the nearest neighbor pyramidal cell, and inhibits the neighboring pyramidal cells. The axon-collaterals of the pyramidal cells in CA1 are sent to various other area such as the subiculum, the enthorinal cortex, and the subcortical areas.

The inhibitory cells in CA3 and CA1 have relatively long axon-collaterals and constitute an interneuronal network $[14,15]$. There exist the collinergic and GABAergic afferents from the septum to the hippocampal CA3 and CA1. Here, the collinergic afferents contact both the pyramidal and inhibitory cells of hippocampus. On the other hand, the GABAergic afferents make synaptic contacts mainly with the inhibitory cells, but not pyramidal cells (for the collinergic afferent, see Frotscher and Leranth [16], and for the GABAergic afferents, see Freund and Antal [17], and Toth et al. [18]). Actually, Toth et al. concluded that their experiments suggest that the GABAergic septohippocampal afferents selectively inhibit the hippocampal inhibitory cells and consequently disinhibit the pyramidal cells.

Taking into account these anatomical and physiological facts, and the hypothetical assertions suggested so far, we construct the network model of the hippocampus. In the following subsections, we treat the model of CA3 network and its dynamics, the CA1 network model, and the overall network model and its dynamics. 


\subsection{A model of the hippocampal CA3 and its dynamics}

Since the network structure and the physiological facts known in CA3 is similar to those factors of the model network for the dynamic association of memories which was proposed by one of the authors [19, 20, 21, 24], we construct a model of CA3, modified the previous model. The neurodynamics we took is as follows.

Let $X$ and $Y$ be a $N$-dimensional vector, whose $i$-th component is denoted by $x_{i}$ and $y_{i}$, respectively. Here, $x_{i}$ and $y_{i}$ are interpreted as the activity of $i$-th pyramidal and inhibitory cells, respectively. Let us introduce a discrete time step $t$ for the development of neurodynamics. The following equation provides $2 N$-dimensional dynamical system composed of the pyramidal and inhibitory cell dynamics.

$$
(X(t+1), Y(t+1))=F(X(t), Y(t)),
$$

where $F$ is $2 N$ nonlinear transformations denoting neurodynamics. Taking into account the effect of the stimulus-induced stochastic release of synaptic vesicles widely observed in the hippocampus, however, we here interpret $F$ as representing a stochatic renewal of neurodynamics, as in the case of Tsuda's model of dynamic associative memories [19, 20, 21, 24].

The stochastic renewal of neurodynamics for the $i$-th set of the pyramidal and the inhibitory cells is defined as follows.

$$
\begin{aligned}
F_{i}(X, Y) & =\left(f_{x}(X, Y), f_{y}(X)\right), \\
x_{i}(t+1) & =f_{x}(X(t), Y(t)), \\
& = \begin{cases}H_{x}\left(\frac{1}{N} \sum_{j=1}^{N} w_{i j} x_{j}-d_{i} y_{i}\right) & \text { (wih probability } \left.p_{x}\right) \\
x_{i}(t) & \text { (otherwise) }\end{cases} \\
y_{i}(t+1) & f_{y}(X(t)), \\
& = \begin{cases}H_{y}\left(\frac{1}{N} \sum_{j=1}^{N} e_{i j} x_{j}\right) & \text { (with probability } \left.p_{y}\right) \\
0 & \text { (otherwise) }\end{cases}
\end{aligned}
$$


where $x_{i} \in[-1,1](i=1, \cdots, N), y_{i} \in[-1,1](i=1, \cdots, N), w_{i j}$ denotes a synaptic weight from pyramidal cell $j$ to $i$, whose concrete form is given below, $e_{i j} \in[0, \alpha]$ a synaptic weight from the pyramidal cell $j$ to the inhibitory cell $i$, and $d_{i} \in[0, \beta]$ a synaptic weight from the inhibitory cell $i$ to the pyramidal cell $i$. Furthermore, as mentioned above, we use a sigmoid function as follows.

$$
H_{x}(z)=H_{y}(z)=2 /(1+\exp (-\gamma z))-1 .
$$

Here $\gamma$ is a steepness parameter.

Memories here are represented by a vector, each component of which denotes the activity of the pyramidal cell. The memories are constructed in the recurrent network of pyramidal cells by the Hebbian learning algorithm

$$
w_{i j}=\frac{1}{K} \sum_{\mu} x_{i}^{\mu} x_{j}^{\mu}
$$

where $x_{i}^{\mu}$ is a $i$-th component of the $\mu$-th memory, $X^{\mu}=\left(x_{1}^{\mu}, \cdots, x_{N}^{\mu}\right)$. It should be noted that the Hebbian algorithm insures a complete retrieval of memory, that is, $X=W X$ for the connection matrix $W$ constructed by orthonormal patterns $X^{\mu}(\mu=1, \cdots, K)$. We then introduce the correlation index of stored patterns [24]. The definition of the correlation index is as follows.

$$
\text { Cor }=\frac{2}{N K(K-1)} \sum_{\mu} \sum_{\nu>\mu}<X^{\mu}, X^{\nu}>,
$$

where $<A, B>$ denotes an inner product of vectors $A$ and $B$.

Let us first describe the properties of CA3 network without the effects of the inhibitory neurons, namely $d_{i}=0$ for all $i$. This condition of the network can occur in the physiological condition that the inhibitory neurons in CA3 are inhibited by the GABAergic afferents coming from the septum. In this case, as in the conventional model of associative memory, the network dynamics become attractor dynamics. In other words, the phase space 
is decomposed into basins of attraction, each of which is represented by an attractor, say a fixed point attractor. Some attractors are generated by the above Hebbian learning algorithm, thus these represent memories, but others are a parasitic one inevitably produced by the correlations between memories. An initial condition implies an input pattern to the network, and the relaxation process to a ceratin attractor implies the retrieval process of the memory concerned. However, this type of network cannot produce the transitions from one attractor to other attractors. Rather, in this network, quantities such as the parameter dependence of memory capacity, the time duration necessary for the transition, and the probabilities of retrieving memories are a subject to study. The system's parameter here is the probabilities $p_{x}$ and $p_{y}$ with each of which the respective network is renewed its dynamics.

We further describe the properties of the CA3 network in the case without the effects of GABAergic neurons from the septum. This situation is similar to the one in the dynamic associative memory model [19, 20, 21, 24, 2, 22, 23]. In the simulation, we fix the value of each $d_{i}$, randomly choosing a value among the values uniformly distributed on $[0, \beta]$. Expectedly, the chaotic transitions among memories occur. In other words, a chaotic sequence of memory patterns is generated (see Fig. 1). - Fig. 1 -

\section{$3.2 \quad$ A model of CA1}

In this subsection, we provide a model of CA1 network and study its dynamics. Let $U$ and $V$ be a vector of the space $[0,1]^{M}$, writing $U=\left(u_{1}, \cdots, u_{M}\right)$ and $V=\left(v_{1}, \cdots, v_{M}\right)$, where $u_{i}$ denotes the state of pyramidal cell $i$ and $v_{i}$ 
the state of stellate cell $i$. Here, $M$ is the number of cell of each type.

$$
\begin{aligned}
U(t+1) & =G(U(t), X(t)), \\
G_{i}(U, X) & =g_{i}(h(U), X), \\
u_{i}(t+1) & =g_{i}(V(t), X(t)), \\
& =H_{u}\left(\epsilon \frac{1}{N} \sum_{j} T_{i j} \frac{\left(x_{j}^{\prime}(t)+1\right)}{2}-\delta \sum_{j} c_{i j} v_{j}(t)+\theta_{i}\right), \\
v_{i}(t) & =h_{i}(U(t)), \\
& =\sum_{j} b_{i j} u_{j}(t), \\
x_{j}^{\prime}(t) & =x_{j}(t) \quad\left(x_{1}(t)>0\right), \\
x_{j}^{\prime}(t) & =-x_{j}(t) \quad\left(x_{1}(t)<0\right)
\end{aligned}
$$

Here, $T_{i j}$ denotes the synaptic connection from the CA3 pyramidal cell $j$ to the pyramidal cell $i$ in CA1, and its value is fixed to the randomly chosen value among the values uniformly distributed on $[0,1]$. The introduction of the variable $x^{\prime}$ is due to a symmetry of the network. For the two types of connections in CA1, $c_{i j}$ denotes the synaptic connection from the stellate cell $j$ to the pyramidal cell $i$, and $b_{i j}$ the synaptic connection from the pyramidal cell $j$ to the stellate cell $i$. In the present simulation, we treat the case that $b_{i i}=1.0$ and $b_{i j}=0$ if $i \neq j$, and also $c_{i i}=1.0$ and $c_{i j}=0$ if $i \neq j$. In the second equation on $u_{i}$, we express the first term by $\frac{x_{j}^{\prime}(t)+1}{2}$ because of the physiological plausibility that the Shaffer collaterals of the CA3 pyramidal cells are excitatory. Here, we assume $\delta \ll 1$ in the presence of GABAergic inhibitory inputs from the septum to the stellate cells in CA1, and $\delta=O(1)$ in the absence of such disinhibitions.

Furthermore, we use a sigmoid function as an input-output transformation of the pyramidal cells.

$$
H_{u}(z)=1 /\left(1+\exp \left(-\gamma_{u} z\right)\right)
$$


where $\gamma_{u}$ is a steepness parameter.

In the simulation, we determine the parameter values to satisfy the inequality providing the contraction condition.

\section{Cantor coding in the hippocampus}

In chaos-driven contracting dynamics, in general, the information read out by chaotic dynamics is written in the contracting subspace by the contracting dynamics, as is seen in the dissipative baker's transformation. In other words, a symbol sequence created by chaotic dynamics is represented as a subset of a Cantor set generated in the contracting subspace. A code table can thus be formed on a Cantor set. Actually, one-to-one correspondence between each symbol sequence generated by chaotic dynamics and each position of Cantor element has been elucidated in the model of the system of unidirectional coupling from $\mathrm{CA} 3$ to $\mathrm{CA} 1$, i.e, $\mathrm{CA} 3 \rightarrow \mathrm{CA} 1$.

In our framework, the CA3 network is a device for the generation of a sequence of patterns. The existence of such a sequence is insured by the presence of chaotic itinerancy [19, 20, 21, 25, 26]. The distance between (or the closeness of) memories represented by a spatial pattern of neuron activity can be specified in CA3 by a scalar product between each two patterns. On the other hand, the distance between different sequences can be defined in CA1 by the Eucledian norm between corresponding two points in the Cantor set. In fact, it can be made by means of the hierarchies embedded in the Cantor set. We have verified the existence of such a hierarchical coding in the model CA1 network of any temporal sequence given by the stimulations of the Shaffer collaterals (see Fig. 2). We have also verified the existence of hierarchical coding in the model CA1 network when the model CA3 network produces a temporal sequence of patterns linked by chaotic orbits (see Fig. 
3). The details of this study will be published elsewhere (Kuroda and Tsuda 2001).

- Fig. $2-$

- Fig. $3-$

\section{Summary and discussion}

We proposed in this paper dynamical models of temporal coding of information in which chaotic itinerancy and Cantor set attractors are linked in terms of the Cantor coding. The temporal coding was realized by the mechanism that a temporal structure generated by chaotic dynamics is hierarchically embedded into a Cantor set in a contracting subspace. We showed that this type of coding can characterize the information processing in the hippocampus.

We were motivated a mathematical part of the present study by the paper of Hata and Yamaguti [27] which clarified the relation of chaos and fractal, and also by the paper of Rössler et al. [11] which showed a possible dynamical mechanism of the appearance of singular-continuous nowhere-differentiable attractors. The late Professor Yamaguti had influenced one of the authors, I. T. over twenty years by his discernment for applied mathematics as well as his personality. I. T. was actually impressed by his words, "Investigating the scientific subjects in the spirit of applied mathematics, one inevitably studies the fundamental parts of mathematics and will eventually find the close relation to many subjects in other different disciplines of sciences". We believe our way of the present study follows his way of thinking of applied mathematics.

Acknowledgments 
This work is supported by a Grant-in-Aid no. 12210001 for Scientific Research on Priority Areas (C) - Advanced Brain Science Project -, and also no. 12834001 for Basic Research (C) - Science of Complex Systems from Ministry of Education, Science, Sports, and Culture, Japan. 


\section{References}

[1] Fujii, H., Ito, H., Aihara, K., Ichinose, N., and Tsukada, M. Dynamical cell assembly hypothesis - Theoretical possibility of spatio-temporal coding in the cortex. Neural Networks 9 (1996) 1303-1350.

[2] Tsuda,I. Towards an interpretation of dynamic neural activity in terms of chaotic dynamical systems. To be published in Behavioral and Brain Sciences 24 (4), 2001.

[3] Karlin, S. Some random walks arising in learning models I, Pacific Journal of Mathematics 3 (1953) 725-756.

[4] Norman, M. F. Some convergence theorems for stochastic learning models with distance diminishing operators. Journal of Mathematical Psychology 5 (1968) 61-101.

[5] Barnsley, M. Fractals Everywhere. Academic Press, San Diego, CA. 1988.

[6] Bressloff, P. C. and Stark, J. Analysis of associative reinforcement learning in neural networks using iterated function systems. IEEE Transactions on Systems, Man, and Cybernetics 22 (1992) 1348-1360.

[7] Pollack, J. B. The induction of dynamical recognizers. Machine Learning 7 (1991) 227-252.

[8] Elman, J. L. Finding structure in time. Cognitive Science 14 (1990) 179-211.

[9] Elman, J. L. Distributed representations, simple recurrent networks, and grammatical structure. Machine Learning 7 (1991) 195-225. 
[10] Kolen, J. F. Recurrent Networks: State machines or iterated function systems? In: Proceedings of 1993 connectionist models summer school: 203-210. Lawlence Erlbaum Associates, Inc., Hillsdale, NJ. 1994.

[11] Rössler, O. E., Wais, R., and Rössler, R. (1992). Singular-continuous Weierstrass function attractors, In Proceedings of the 2nd International Conference on Fuzzy Logic and Neural Networks, (Iizuka, Japan) 909912.

[12] Tsuda, I. (1996) A new type of self-organization associated with chaotic dynamics in neural systems. International Journal of Neural Systems 7: 451-459.

[13] Tsuda, I. and Yamaguchi, A. (1998) Singular-continuous nowheredifferentiable attractors in neural systems. Neural Networks 11:927-937.

[14] Buszaki, G. Functions for interneuronal nets in the hippocampus. Can. J. Physiol. Pharmacol. 75 (1997) 508-515.

[15] Freund, T. F. and Gulyas, Inhibitory control of GABAergic interneurons in the hippocampus. Can. J. Physiol. Pharmacol. 75 (1997) 479-487.

[16] Frotscher, M. and Leranth, C. Collinergic innervation of the hippocampus as revealed by colline acetyltransferase immunocytochemistry: a combined light and electron microscopic study. Journal of Comparative Neurology 239 (1985) 237-246.

[17] Freund, T. F. and Antal, M. GABA-containing neurons in the septum control inhibitory. Nature 336 (1988) . 
[18] Toth, K., Freund, T. F., and Miles, R. Disinhibition of rat hippocampal pyramidal cells by GABAergic afferents from the septum. Journal of Physiology 500.2 (1997) 463-474.

[19] Tsuda, I., Körner, E., and Shimizu, H. Memory dynamics in asynchronous neural networks. Progress of Theoretical Physics 78 (1987) 51-71.

[20] Tsuda, I. Chaotic itinerancy as a dynamical basis of Hermeneutics of brain and mind. World Futures 32 (1991) 167-185.

[21] Tsuda, I. Chaotic neural networks and thesaurus. In: Neurocomputers and Attention I, ed., A. V. Holden and V. I. Kryukov. Manchester University Press, Manchester, 1991, 405-424.

[22] Aihara, K., Takabe, T., and Toyoda, M. Chaotic neural networks. Physics Letters A 144 (1990) 333-340.

[23] Nara, S. and Davis, P. Chaotic wandering and search in a cycle-memory neural network. Progress of Theoretical Physics 88 (1992) 845-855.

[24] Tsuda, I. Dynamic link of memories-chaotic memory map in nonequilibrium neural networks. Neural Networks 5 (1992) 313-326.

[25] Ikeda, K., Otsuka, K., and Matsumoto, K. (1989) Maxwell-Bloch turbulence, Progress of Theoretical Physics, Supplement, 99: 295-324.

[26] Kaneko, K. (1990) Clustering, coding, switching, hierarchical ordering, and control in network of chaotic elements. Physica D 41: 137-172.

[27] Hata, M. and Yamaguti, M. Takagi function and its generalization, Japan Journal of Applied Mathematics, 1 (1984) 186-199. 


\section{Figure Captions}

Fig.1 Time series of neural activity in CA3. The neural activity is represented by the overlapping $m^{(\mu)}(t)=\left|\left(x(t), X^{(\mu)}\right)\right|$ for $\mu=1,2,3,4$, namely an inner product of the activity of pyramidal cells and the $\mu$-th stored pattern. Four time series are overlaid. The value $m^{(\mu)}=1$ indicates a complete retrieval of memories. The figure shows a successive retrieval of memories. The parameter values for this simulation: $N=32, K=4, \beta=1.0, p_{x}=$ $0.6, p_{y}=1.0$.

Fig.2 A Cantor coding in phase space constructed by the membrane potential of CA1 pyramidal neurons. The case with the inputs of random sequence consisting of three almost orthogonal patterns. The patterns are denoted by the number 1, 2 and 3. The label $\mathrm{nm}$ denotes the temporal patern sequence from $m$ to $n$. The most left cluster indicates all the pattern sequences of the "present" pattern 2, the right lower one for the "present" pattern 3, and the right upper one for the "present" pattern 1. Each cluster consists of further three subclusters which indicate the "previous" patterns appeared at one time step before in the sequence. The itinerary of a random sequence is thus hierarchically represented in the self-similar structure of a Cantor set.

Fig.3 A Cantor coding in phase space constructed by the membrane potential of CA1 pyramidal neurons. The case with the inputs from CA3 chaotic outputs. Memories in CA3 are here represented by three almost orthogonalized patterns, $X^{(1)}, X^{(2)}$, and $X^{(3)}$. Here we supposed a periodic repetition of inhibition and disinhibition with a period 4 (time-steps) which may stem from the septum. The figure shows a certain particular part of activity of CA1 at time $t+1$ when the CA3 activity $X(t)$ equals to the activity for the memory $3, X^{(3)}$. Three symbols in the figure indicate each particular tran- 
sition to a memory pattern $X^{(3)}$ from a respective following pattern after four time-steps: a cross denotes the transition from $X^{(1)}$, a plus from $X^{(2)}$, and an asterisk from $X^{(3)}$. We further adjusted a threshold $\theta$ in CA1 model such that it has a slightly positive value when the inhibitory inputs from the septum are absent and zero when such inputs are present. 


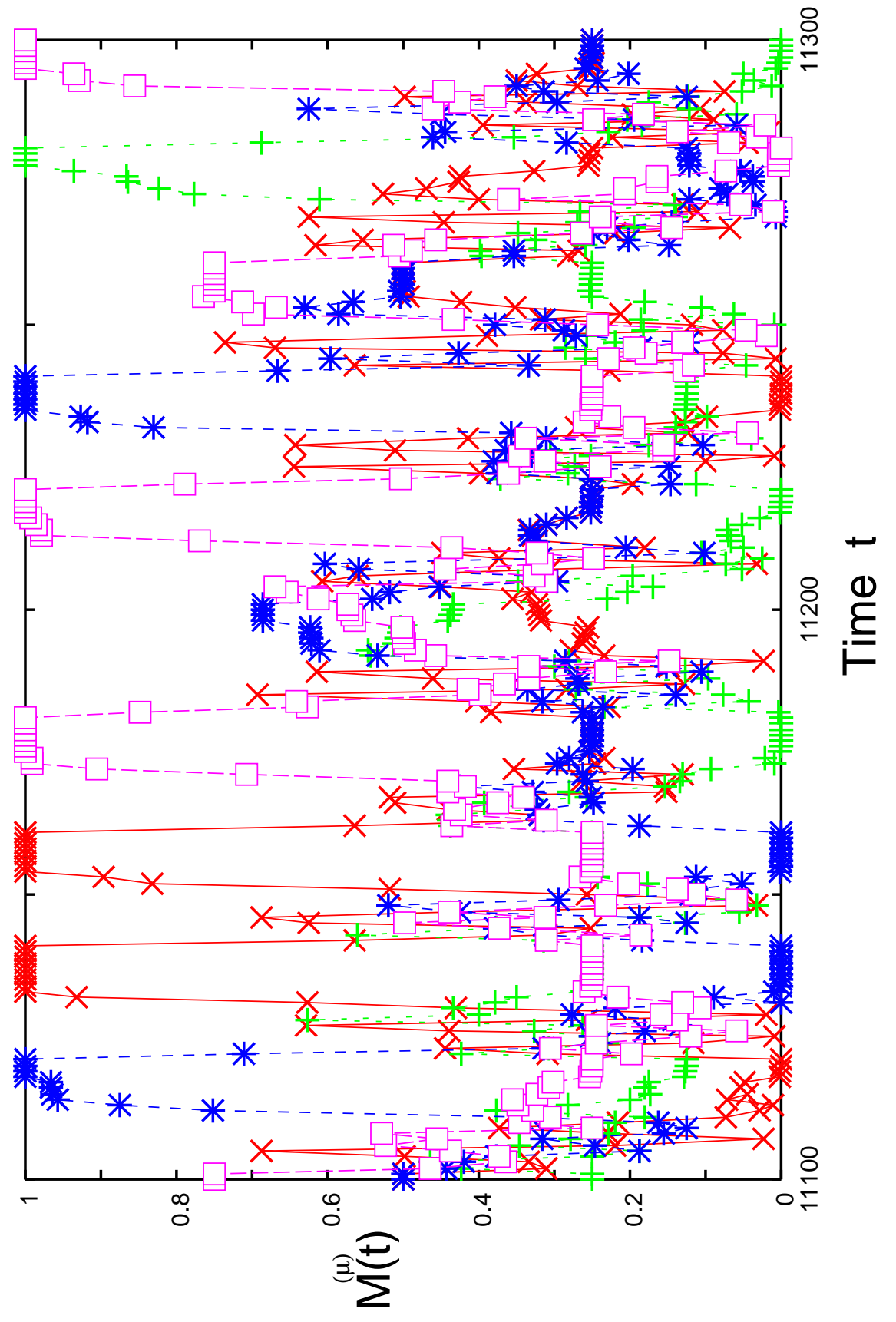

Figure 1: 


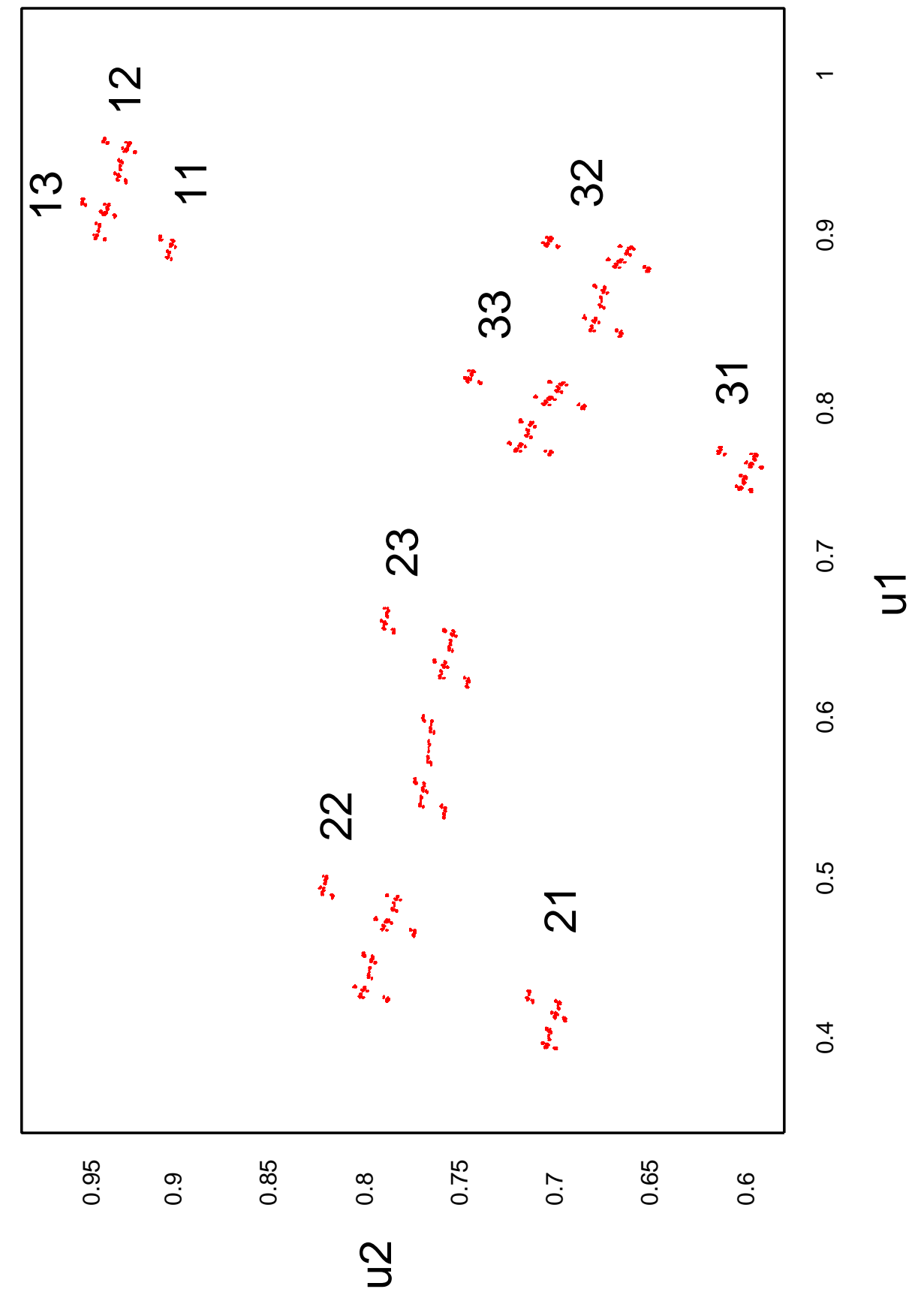

Figure 2: 


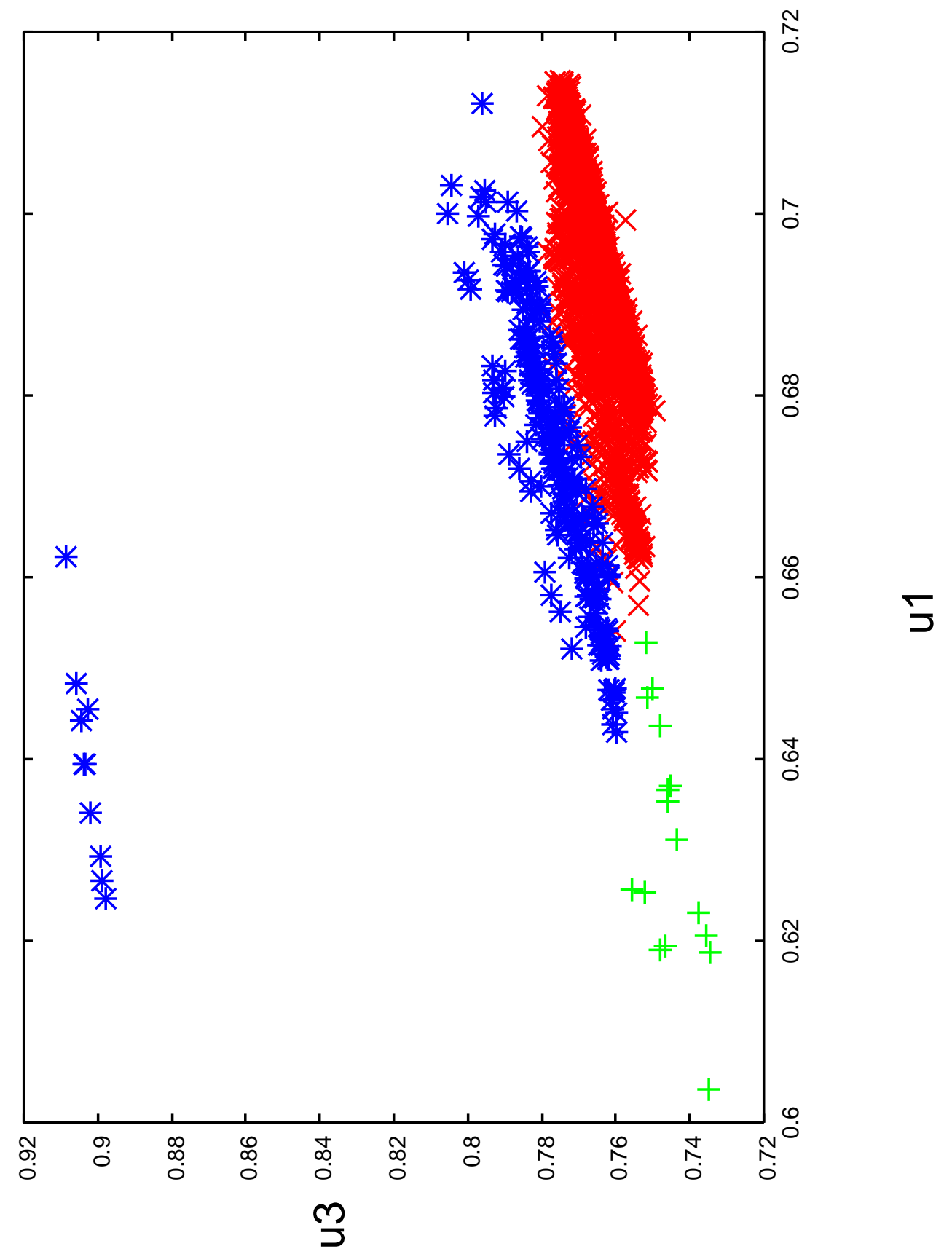

Figure 3: 